\title{
Improved Family of Ratio Estimators of Finite Population Variance in Stratified Random Sampling
}

\author{
Etebong P C* \\ Department of Mathematics and Statistics, University of Uyo, Nigeria
}

Submission: December 19, 2017; Published: February 23, 2018

*Corresponding author: Etebong P Clement, Department of Mathematics and Statistics, University of Uyo, Nigeria, Email: epclement@yahoo.com

\begin{abstract}
This paper introduces a new family of exponential ratio estimators of population variance in stratified random sampling and studies its properties. Based on Bahl \& Tuteja [1], Kadillar \& Cingi [2] and Solanki et al. [3], membership of the new family of estimators is identified. Analytical and numerical results show that under certain prescribed conditions, the new estimator has equal optimal efficiency with the regression estimator of population variance but always fares better than the classical ratio estimator of population variance by Isaki [4] and every identified existing estimator of its family.
\end{abstract}

Keywords: Optimum estimator; Large sample approximation; Optimal efficiency; Regression estimator

\section{Introduction}

In sampling theory population information of the auxiliary variable such as the total, mean and variance are often used to provide additional information which help to increase the efficiency of the estimation of the population parameter(s) of interest. When the information on an auxiliary variable is known, one can use the ratio, product and regression estimators to improve the performance of the estimator of the study variable. When the correlation between the study variable and the auxiliary variable is positive, ratio method of estimation is quite effective. Many authors like [5-19] among others have proposed different ratio estimators (of total or mean) in sample surveys.

The estimation of the finite population variance has been of great significance in various fields such as Industry, Agriculture, Medical and Biological sciences. Various authors such as [4,2035 ] have used auxiliary information to improve the efficiency of the estimator of population variance of the study variable. In this paper, the problem of constructing efficient estimator of the population variance is considered and a new family of exponential ratio estimators of population variance in stratified random sampling that is as efficient as the general regression estimator is introduced.

\section{Basic notations and definitions}

Consider a finite population $\Pi=\left(\pi_{1}, \pi_{2}, \ldots, \pi_{N}\right)$ of size $(N)$. Let $(X)$ and $(Y)$ denote the auxiliary and study variables taking values $X_{i}$ and $Y_{i}$ respectively on the $i$-th unit $\pi_{i}(i=1,2, \ldots, N)$ of the population. Let the population be divided into $K$ strata with $N_{k}$ units in the $k^{\text {th }}$ stratum from which a simple random sample of size $n_{k}$ is taken without replacement. The total population size be $N=\sum_{k=1}^{K} N_{k}$ and the sample size $n=\sum_{k=1}^{K} n_{k}$, respectively. Associated with the $i$ th element of the $h^{\text {th }}$ stratum are $y_{k i}$ and $x_{k i}$ with $x_{k i}>0$ being the covariate; where $y_{h i}$ is the $y$ value of the ith element in stratum $k$, and $x_{k i}$ is the $x$ value of the ith element in stratum $k, k=1,2, \ldots, K$, and $i=1,2, \ldots, N_{k}$. For the $k^{\text {th }}$ stratum, let $W_{k}=N_{k} / N$ be the stratum weights and $f_{k}=n_{k} / N_{k}$ the sample fraction. Let the $k^{\text {th }}$ stratum means of the study variable $Y$ and auxiliary variable $X\left(\bar{y}_{k}=\Sigma_{i=1}^{n_{k}} y_{k i} / n_{k} ; \bar{x}_{k}=\Sigma_{i=1}^{n_{k}} x_{k i} / n_{k}\right)$ be the unbiased estimator of the population stratum means $\left(\bar{Y}_{k}=\sum_{i=1}^{N_{k}} y_{k i} / N_{k} ; \bar{X}_{k}=\sum_{i=1}^{N_{k}} x_{k i} / N_{k}\right)$ of $Y$ and $X$ respectively, based on $n_{k}$ observations.

Let,

$$
s_{k y}^{2}=S_{k y}^{2}\left(1+e_{k S_{y}}\right), s_{k x}^{2}=S_{k x}^{2}\left(1+e_{k S_{x}}\right)
$$

So that,

$E\left(e_{k S_{x}}\right)=E\left(e_{k S_{y}}\right)=0, E\left(e_{k S_{x}}^{2}\right)=\gamma_{k}\left[\beta_{2}(x)-1\right], E\left(e_{k S_{x}} e_{k S_{y}}\right)=\gamma_{k}\left(\lambda_{22}-1\right)$,

Where,

$\gamma_{k}=\left(\frac{1-f_{k}}{n_{k}}\right)=\left(\frac{1}{n_{k}}-\frac{1}{N_{k}}\right) ; S_{k x}^{2}=\frac{1}{N_{k}-1} \sum_{i=1}^{N_{k}}\left(x_{k i}-\bar{X}_{k}\right)^{2} ; S_{k y}^{2}=\frac{1}{N_{k}-1} \sum_{i=1}^{N_{k}}\left(y_{k i}-\bar{Y}_{k}\right)^{2}$

$S_{k x y}=\frac{1}{N_{k-1}} \sum_{i=1}^{N_{k}}\left(x_{k i}-\bar{X}_{k}\right)\left(y_{k i}-\bar{Y}_{k}\right)$,

$\beta_{2 k}(y)=\left[\mu_{k 40}(y, y) / \mu_{k 20}^{2}(y, y)\right]$,

$\beta_{2 k}(x)=\left[\mu_{k 40}(x, x) / \mu_{k 20}^{2}(x, x)\right]$,

$\lambda_{y x}=\left[\mu_{k 22}(y, x) / \mu_{k 20}(y, x)\right]$, 


$$
\begin{aligned}
& \mu_{k r s}(y, x)=N_{k}^{-1} \sum_{i=1}^{N_{k}}\left(y_{k i}-\bar{Y}_{k i}\right)^{r}\left(x_{k i}-\bar{X}_{k i}\right)^{s}, \\
& \grave{U}=\left[\left(\lambda_{22}-1\right) /\left[\beta_{2}(x)-1\right]\right] \\
& \rho_{k}=\left(\lambda_{22}-1\right)\left(\left[\beta_{2 k}(x)-1\right]\left[\beta_{2 k}(y)-1\right]\right)^{-\frac{1}{2}}
\end{aligned}
$$

And,

$$
\psi=S_{k y}^{2} / S_{k x}^{2}
$$

\section{The suggested estimator}

The suggested exponential ratio estimator of population variance is given by:

$$
S_{p r}^{2}=\sum_{k=1}^{K} W_{k} s_{k y}^{2}\left\{\tau_{k}-\alpha_{k}\left(\frac{s_{h x}^{2}}{S_{k x}^{2}}\right)^{\eta_{k}} \exp \left[\frac{\omega_{k}\left(s_{k x}^{2}-S_{k x}^{2}\right)}{\left(s_{k x}^{2}+S_{k x}^{2}\right)}\right]\right\}
$$

Where $\alpha_{k}, \tau_{k}$ and $\eta_{k}$ are suitably chosen scalars such that $\alpha_{k}$ and $\tau_{k}$ satisfies the condition $\tau_{k}=1+\alpha_{k} ;-\infty \leq \alpha_{k} \leq \infty$. Expressing (1) in terms of the $e^{\prime} s$ gives

$S_{p r}^{2}=\sum_{k=1}^{K} W_{k} S_{k y}^{2}\left(1+e_{k S_{y}}\right)\left\{\tau_{k}-\alpha_{k}\left(1+e_{k S_{x}}\right)^{\eta_{k}} \exp \frac{\omega_{k} e_{k S_{x}}}{2}\left(1+\frac{1}{2} e_{k S_{x}}\right)^{-1}\right\}$

Now, it is assumed that $\left|e_{k S_{x}}\right|<1, \quad\left|e_{k S_{y}}\right|<1$ so that expanding $\left(1+e_{k S_{x}}\right)^{\eta_{k}},\left(1+\frac{1}{2} e_{k S_{x}}\right)^{-1}$ and $\exp \frac{\omega_{k} e_{k S_{x}}}{2}\left(1+\frac{1}{2} e_{k S_{x}}\right)^{-1}$ as a series in power of $e_{k s_{x}}$, multiplying out and retaining terms of the $e^{\prime} s$ to the second degree gives

$\hat{S}_{p r}^{2}=\sum_{k=1}^{K} W_{k} S_{k y}^{2}\left[1-\frac{\alpha_{k}\left(2 \eta_{k}+\omega_{k}\right) e_{k S_{x}}}{2}-\alpha_{k}\left(2 \eta_{k}+\omega_{k}\right) \frac{\left(2 \eta_{k}+\omega_{k}-2\right) e_{k S_{x}}^{2}}{8}-\alpha_{k}\left(2 \eta_{k}+\omega_{k}\right) e_{k S_{x}} e_{k S_{y}}+e_{k S_{y}}\right]$

So that

$$
\left(S_{p r}^{2}-S_{y}^{2}\right)=\sum_{k=1}^{K} W_{k} S_{k y}^{2}\left[\left\{-\frac{\alpha_{k}\left(2 \eta_{k}+\omega_{k}\right)}{2}\left(e_{k S_{x}}+\frac{\left(2 \eta_{k}+\omega_{k}-2\right) e_{k S_{x}}^{2}}{4}+e_{k S_{x}} e_{k S_{y}}\right)\right\}+e_{k S_{x}}\right]
$$

Taking the expectation of (2), the Bias of estimator $S_{p r}^{2}$ is obtained as:

$B\left(S_{p r}^{2}\right)=\sum_{k=1}^{K} W_{k} \gamma_{k} S_{k y}^{2}\left[-\frac{\alpha_{k}\left(2 \eta_{k}+\omega_{k}\right)}{2}\left(\frac{1}{4}\left(2 \eta_{k}+\omega_{k}-2\right)\left[\beta_{2}(x)-1\right]+\left(\lambda_{22}-1\right)\right)\right]$ (3)

Squaring both sides of (2) and taking expectation, the Mean Square Error is obtained by Taylor's series approximation as:

$$
S E\left(S_{p r}^{2}\right)=\sum_{k=1}^{K} W_{k}^{2} \gamma_{k} S_{k y}^{4}\left[\left[\beta_{2}(y)-1\right]+\frac{\alpha_{k}\left(2 \eta_{k}+\omega_{k}\right)}{4}\left(\alpha_{k}\left(2 \eta_{k}+\omega_{k}-2\right)\left[\beta_{2}(x)-1\right]-4\left(\lambda_{22}-1\right)\right)\right]
$$

The suggested estimator $S_{p r}^{2}$ attains its optimal efficiency if $\left(\eta_{k}, \omega_{k}\right)=(1,1)$ and $\frac{\partial M S E\left(S_{p r}^{2}\right)}{\partial \alpha_{k}}=0$

So that

$$
\begin{aligned}
& \alpha_{k}=\frac{2 \Omega}{3} \\
& =\alpha_{k, o p t}
\end{aligned}
$$

Substituting the value of ${ }^{\alpha_{k, o p t}}$ in (5) for $\alpha_{k}$ in (4), gives the MSE of an asymptotically optimum exponential ratio estimator (AOE) $S_{p r}^{2}$ (or minimum MSE of $S_{p r}^{2}$ ) as:

$$
M S E_{o p t}\left(S_{p r}^{2}\right)=\sum_{k=1}^{K} W_{k}^{2} S_{k y}^{4} \gamma_{k}\left[\beta_{2}(y)-1\right]\left(1-\rho_{k}^{2}\right)(6)
$$

\section{Alternatively}

The suggested estimator $S_{p r}^{2}$ would attain its optimal efficiency if:

$$
\frac{\partial M S E\left(S_{p r}^{2}\right)}{\partial \omega_{k}}=\frac{\partial M S E\left(S_{p r}^{2}\right)}{\partial \eta_{k}}=\frac{\partial M S E\left(S_{p r}^{2}\right)}{\partial \alpha_{k}}=0
$$

So that

$$
\begin{aligned}
& \alpha_{k}=\frac{2\left(\lambda_{22}-1\right)}{\left(2 \eta_{k}+\omega_{k}\right)\left[\beta_{2}(x)-1\right]} \\
& \eta_{k}=\frac{2\left(\lambda_{22}-1\right)-\omega_{k} \alpha_{k}\left[\beta_{2}(x)-1\right]}{2 \alpha_{k}\left[\beta_{2}(x)-1\right]} \\
& \omega_{k}=\frac{2\left\{\left(\lambda_{22}-1\right)-\eta_{k} \alpha_{k}\left[\beta_{2}(x)-1\right]\right\}}{\alpha_{k}\left[\beta_{2}(x)-1\right]}
\end{aligned}
$$

Remark 1: Following from ((7), (8), (9)), it should be noted here that the proposed estimator $S_{p r}^{2}$ would also attain its optimal efficiency with minimum MSE given as in (6), when either of the following optimality conditions is satisfied:

(i) $\eta_{k, o p t}=\eta_{k}, \omega_{k, \text { opt }}=\omega_{k}$ and $\alpha_{k, o p t}=\left(\frac{2 \Omega}{2 \eta_{k}+\omega_{k}}\right)$

(ii) $\eta_{k, o p t}=\eta_{k}, \alpha_{k, o p t}=\alpha_{k}$ and $\omega_{k, o p t}=\frac{2\left[\Omega-\eta_{k} \alpha_{k}\right]}{\alpha_{k}}$

(iii) $\omega_{k, \text { opt }}=\omega_{k}, \alpha_{k, o p t}=\alpha_{k}$ and $\eta_{k, o p t}=\left(\frac{2 \Omega-\omega_{k} \alpha_{k}}{2 \alpha_{k}}\right)$

\section{Membership of the proposed estimator}

This section studies the properties of the proposed estimator and identifies some special members of its family and derives their mean square errors (MSEs) under certain prescribed conditions.

\section{Stratified random sampling estimator}

When $\tau_{k}=1, \alpha_{k}=0, \quad \eta_{k}=\eta_{k}$ and $\omega_{k}=\omega_{k}$, the resulting family member of the proposed estimator is an unbiased stratified random sampling estimator of population variance given as:

$$
S_{p r 1}^{2}=\sum_{h=1}^{H} w_{h} s_{k y}^{2}
$$

With MSE given as

$$
\operatorname{MSE}\left(S_{p r 1}^{2}\right)=\sum_{k=1}^{K} W_{k}^{2} \gamma_{k} S_{k y}^{4}\left[\beta_{2}(y)-1\right]
$$

Remark 2: It should be noted here that irrespective of the values of $\eta_{k}$ and $\omega_{k}$, whenever $\tau_{k}=1$ and $\alpha_{k}=0$; the resulting family member of the proposed estimator is always the unbiased stratified random sampling estimator of population variance given in (10).

\section{Bahl \& Tuteja [1] exponential ratio-type estimator}

When $\tau_{k}=0, \alpha_{k}=-1, \quad \eta_{k}=0$ and $\omega_{k}=-1$, the resulting family member of the proposed estimator is the Bahl and Tuteja (1991) 
exponential ratio-type estimator of population variance in stratified random sampling given as:

$$
S_{p r 2}^{2}=\sum_{k=1}^{K} W_{k} s_{k y}^{2} \exp \left[\frac{\left(S_{k x}^{2}-S_{k x}^{2}\right)}{\left(S_{k x}^{2}+S_{k x}^{2}\right)}\right]
$$

with MSE given as:

$$
\operatorname{MSE}\left(S_{p r 2}^{2}\right)=\sum_{k=1}^{K} W_{k}^{2} \gamma_{k} S_{k y}^{4}\left[\left[\beta_{2}(y)-1\right]+\frac{1}{4}\left(\left[\beta_{2}(x)-1\right]-4\left(\lambda_{22}-1\right)\right)\right]
$$

\section{Kadilar \& Cingi [2] chain ratio-type estimator}

When $\tau_{k}=0, \alpha_{k}=-1, \eta_{k}=-2$ and $\omega_{k}=0$, the resulting family member of the proposed estimator is the Kadilar and Cingi (2003) chain ratio-type estimator of population variance in stratified random sampling given as:

with MSE given as

$$
S_{p r 3}^{2}=\sum_{k=1}^{K} W_{k} s_{k y}^{2}\left(\frac{S_{h x}^{2}}{s_{k x}^{2}}\right)^{2}
$$

$\operatorname{MSE}\left(S_{p r 3}^{2}\right)=\sum_{k=1}^{K} W_{k}^{2} \gamma_{k} S_{k y}^{4}\left[\left[\beta_{2}(y)-1\right]+4\left[\beta_{2}(x)-1\right]-\left(\lambda_{22}-1\right)\right]$

\section{Solanki et al. [3] class of ratio-type estimators}

When $\tau_{k}=2, \alpha_{k}=1, \eta_{k}=\eta_{k}$ and $\omega_{k}=\omega_{k}$, the resulting family member of the proposed estimator is the Solanki et al. [3] class of ratio-type estimators of population variance in stratified random sampling given as:

$$
S_{p r 2}^{2}=\sum_{k=1}^{K} W_{k} s_{k y}^{2}\left\{2-\left(\frac{s_{h x}^{2}}{S_{k x}^{2}}\right)^{\eta_{k}} \exp \left[\frac{\omega_{k}\left(s_{k x}^{2}-S_{k x}^{2}\right)}{\left(S_{k x}^{2}+s_{k x}^{2}\right)}\right]\right\}
$$

With MSE given as

$$
\operatorname{MSE}\left(S_{p r 4}^{2}\right)=\sum_{k=1}^{K} W_{k}^{2} \gamma_{k} S_{k y}^{4}\left[\left[\beta_{2}(y)-1\right]+\left[\left(2 \eta_{k}+\omega_{k}\right) / 4\right] \times\left(\left(2 \eta_{k}+\omega_{k}\right)\left[\beta_{2}(x)-1\right]-4\left(\lambda_{22}-1\right)\right)\right]
$$

\section{Analytical study}

\section{Efficiency comparisons}

This section compares the optimal MSE of the proposed ratio estimator of population variance $\left(S_{p r}^{2}\right)$ with the MSEs of some existing estimators of its family.

\section{Regression estimator}

The proposed ratio estimator would be more efficient than the general regression estimator of population variance $\left(S_{R E G}^{2}\right)$ if:

But

$$
M S E_{o p t}\left(S_{p r}^{2}\right)-M S E_{o p t}\left(S_{R E G}^{2}\right)<0
$$

$$
S_{R E G}^{2}=s_{y}^{2}+\hat{\mathrm{A}}\left(S_{x}^{2}-s_{x}^{2}\right)
$$

Where,
$S_{y}^{2}=\sum_{k=1}^{K} W_{k} S_{k y}^{2}, S_{y}^{2}=\sum_{k=1}^{K} W_{k} s_{k y}^{2}, S_{x}^{2}=\sum_{k=1}^{K} W_{k} S_{k y}^{2}, S_{x}^{2}=\sum_{k=1}^{K} W_{k} s_{k y}^{2}$ So that (18) becomes

$$
S_{R E G}^{2}=\sum_{k=1}^{K} W_{k} s_{k y}^{2}+\mathrm{B} \sum_{k=1}^{K} W_{k}\left(S_{k x}^{2}-s_{k x}^{2}\right)
$$

Expressing (19) in terms of the $e^{\prime} s$ gives

$$
S_{R E G}^{2}=\sum_{k=1}^{K} W_{k} S_{k y}^{2}\left(1+e_{k S_{y}}\right)+\mathrm{B} \sum_{k=1}^{K} W_{k} S_{k x}^{2} e_{k S_{x}}
$$

Now, it is assumed that $\left|e_{k S_{x}}\right|<1$ and $\left|e_{k S_{y}}\right|<1$ so that

$$
\left(S_{R E G}^{2}-S_{y}^{2}\right)^{2}=\sum_{k=1}^{K} W_{k}^{2}\left[S_{k y}^{4} e_{k S_{y}}+\mathrm{B}^{2} S_{k x}^{4} e_{k S_{x}}-2 \mathrm{~B} S_{k y}^{2} S_{k x}^{2} e_{k S_{y}} e_{k S_{x}}\right]
$$

Taking the expectation of (20), the Mean Square Error is obtained by Taylor's series approximation as:

$$
\operatorname{MSE}\left(S_{R E G}^{2}\right)=\sum_{k=1}^{K} W_{k}^{2} \gamma_{k}\left[S_{k y}^{4}\left[\beta_{2 k}(y)-1\right]+\mathrm{B}^{2} S_{k x}^{2}\left[\beta_{2 k}(x)-1\right]-2 \mathrm{~B} S_{k y}^{2} S_{k x}^{2}\left(\lambda_{22 k}-1\right)\right]
$$

Where the value of $B$ that minimizes (21) is given by

$$
\mathrm{B}_{\mathrm{opt}}=\psi \Omega(22)
$$

Substituting the value of $\mathrm{B}_{\text {opt }}$ in (22) for B in (21), gives the optimal MSE $\left(M S E_{o p t}\right)$ of the general regression estimator $\left(S_{R E G}^{2}\right)$ as:

$$
M S E_{o p t}\left(S_{R E G}^{2}\right)=\sum_{k=1}^{K} W_{k}^{2} S_{k y}^{4} \gamma_{k}\left[\beta_{2}(y)-1\right]\left(1-\rho_{k}^{2}\right)
$$

Remark 3: Following from (6) and (23), it is evidenced that under certain prescribed optimality conditions, the proposed ratio estimator $\left(S_{p r}^{2}\right)$ has equal optimal efficiency with the general regression estimator $\left(S^{2}{ }_{R E G}\right)$. The implication is that the proposed exponential ratio estimator of population variance is as good as the general regression estimator of population variance.

\section{Stratified random sampling estimator}

The proposed estimator would be more efficient than the stratified random sampling estimator of population variance if:

$$
\begin{aligned}
& \operatorname{MSE}_{o p t}\left(S_{p r}^{2}\right)-\operatorname{MSE}\left(S_{p r 1}^{2}\right)<0 \\
& \Rightarrow \sum_{k=1}^{K} W_{k}^{2} \gamma_{k} S_{k y}^{4}\left(\lambda_{22 k}-1\right)^{2}\left[\beta_{2}(x)-1\right]^{-1}>0 \\
& \text { Certainly, for (24) to hold } \beta_{2}(x)>1
\end{aligned}
$$

\section{Bahl \& Tuteja [1] exponential ratio-type estimator}

The proposed estimator would be more efficient than the Bahl \& Tuteja [1] exponential ratio-type estimator of population variance if:

$$
\operatorname{MSE} E_{o p t}\left(S_{p r}^{2}\right)-\operatorname{MSE}\left(S_{p r 2}^{2}\right)<0
$$




$$
\Rightarrow \sum_{k=1}^{K} W_{k}^{2} \gamma_{k} S_{k y}^{4}\left(\beta_{2}(x)-2 \lambda_{22 k}+1\right)^{2}>0
$$

So that either

$$
\begin{array}{cl}
\text { (i) } & \sum_{k=1}^{K} W_{k}^{2} \gamma_{k} S_{k y}^{4} \beta_{2}(x)>\sum_{k=1}^{K} W_{k}^{2} \gamma_{k} S_{k y}^{4}\left(2 \lambda_{22 k}-1\right) \\
\text { Or } & \sum_{k=1}^{K} W_{k}^{2} \gamma_{k} S_{k y}^{4} \lambda_{22 k}<\frac{1}{2} \sum_{k=1}^{K} W_{k}^{2} \gamma_{k} S_{k y}^{4}\left[\beta_{2}(x)+1\right] \\
\text { (ii) }
\end{array}
$$

\section{Kadilar \& Cingi [2] chain ratio-type estimator}

The proposed estimator would be more efficient than the Kadilar \& Cingi [2] chain ratio-type estimator of population variance if:

$$
\begin{aligned}
& M S E_{o p t}\left(S_{p r}^{2}\right)-\operatorname{MSE}\left(S_{p r 3}^{2}\right)<0 \\
& \Rightarrow \sum_{k=1}^{K} W_{k}^{2} \gamma_{k} S_{k y}^{4}\left(2 \beta_{2}(x)-\lambda_{22 k}-1\right)^{2}>0
\end{aligned}
$$

So that either

$$
\begin{aligned}
& \text { (i) } \sum_{k=1}^{K} W_{k}^{2} \gamma_{k} S_{k y}^{4} \beta_{2}(x)>\frac{1}{2} \sum_{k=1}^{K} W_{k}^{2} \gamma_{k} S_{k y}^{4}\left(\lambda_{22 k}+1\right) \\
& \text { Or } \\
& \text { (ii) } \sum_{k=1}^{K} W_{k}^{2} \gamma_{k} S_{k y}^{4} \lambda_{22 k}<\sum_{k=1}^{K} W_{k}^{2} \gamma_{k} S_{k y}^{4}\left[2 \beta_{2}(x)-1\right]
\end{aligned}
$$

\section{Solanki et al. [3] class of ratio-type estimators}

\begin{tabular}{|c|c|c|c|c|c|}
\hline Parameter & Stratum 1 & Stratum 2 & Stratum 3 & Stratum 4 & Stratum 5 \\
\hline$N_{k}$ & 40 & 20 & 32 & 26 & 42 \\
\hline$n_{k}$ & 14 & 8 & 10 & 12 & 16 \\
\hline $\bar{X}_{k}$ & 24.62 & 48.63 & 32.85 & 64.36 & 41.43 \\
\hline $\bar{Y}_{k}$ & 35.26 & 42.08 & 16.49 & 22.33 & 36.45 \\
\hline$\beta_{2 k}(y)$ & 21.28 & 35.32 & 18.45 & 42.83 & 28.37 \\
\hline$\beta_{2 k}(x)$ & 10.25 & 16.82 & 22.42 & 12.06 & 18.67 \\
\hline$\lambda_{22 k}$ & 8.86 & 18.56 & 16.2 & 14.25 & 10.17 \\
\hline$S_{k x}^{2}$ & 90.25 & 64.83 & 105.38 & 86.44 & 58.38 \\
\hline$S_{k y}^{2}$ & 125.26 & 82.08 & 78.48 & 92.06 & 112.42 \\
\hline$W_{k}^{2}$ & 0.06 & 0.02 & 0.04 & 0.03 & 0.07 \\
\hline$\gamma_{k}$ & 0.05 & 0.08 & 0.07 & 0.05 & 0.04 \\
\hline$\eta_{k}$ & 1 & -2 & -1 & 2 & 1 \\
\hline$\omega_{k}$ & -1 & 2 & 1 & -2 & -2 \\
\hline
\end{tabular}

The proposed estimator would be more efficient than the Kadilar \& Cingi [2] chain ratio-type estimator of population variance if:

$$
M S E_{o p t}\left(S_{p r}^{2}\right)-\operatorname{MSE}\left(S_{p r 4}^{2}\right)<0
$$

$\Rightarrow \sum_{k=1}^{K} W_{k}^{2} \gamma_{k} S_{k y}^{4}\left(3 \beta_{2}(x)-2 \lambda_{22 k}-1\right)^{2}>0:\left(\eta_{k}, \omega_{k}\right)=(1,1)$

\section{Empirical study}

Table 1: Data Statistics.

\section{Isaki [4] ratio-type estimator}

The proposed estimator would be more efficient than the Isaki [4] ratio-type estimator of population variance if:

$$
\begin{aligned}
& M S E_{\text {opt }}\left(S_{p r}^{2}\right)-\operatorname{MSE}\left(S_{\text {Ratio }}^{2}\right)<0 \\
& \Rightarrow \sum_{k=1}^{K} W_{k}^{2} \gamma_{k} S_{k y}^{4}\left(\beta_{2}(x)-\lambda_{22 k}\right)^{2}>0
\end{aligned}
$$

So that

$$
\sum_{k=1}^{K} W_{k}^{2} \gamma_{k} S_{k y}^{4} \beta_{2}(x)>\sum_{k=1}^{K} W_{k}^{2} \gamma_{k} S_{k y}^{4} \lambda_{22 k}
$$

\section{The percent relative efficiency $(P R E)$}

The percent relative efficiency $(P R E)$ of an estimator $\varphi$ with respect to the stratified random sampling estimator of population variance $\left(S_{p r 1}^{2}\right)$ is defined by:

$$
\begin{aligned}
& \operatorname{PRE}\left(\ddot{\mathrm{o}}, S_{p r 1}^{2}\right)=\frac{\operatorname{MSE}\left(S_{p r 1}^{2}\right)}{\operatorname{MSE}(\varphi)} \times 100 \\
& \operatorname{MSE}\left(S_{p r 1}^{2}\right)=\sum_{k=1}^{K} W_{k}^{2} \gamma_{k} S_{k y}^{4}\left[\beta_{2}(y)-1\right]=3,125.78
\end{aligned}
$$


In this section, the performance of the proposed ratio estimator is assessed with every identified existing estimators of its family and the classical ratio estimator of population variance by Isaki [4]. The merits of the suggested ratio estimator over other existing estimators were judged using the Data Statistics in Table 1.

\section{Discussion of Results}

Analytical comparisons showed that the proposed ratio estimator of population variance under certain realistic conditions is more efficient than the unbiased stratified sampling estimator of population variance, classical ratio estimator of population variance by Isaki [4], Bahl \& Tuteja [1] ratio-type estimator, Solanki et al [3] ratio-type estimator and Kadillar \& Cingi [2] ratio-type estimator but has equal optimal efficiency with the regression estimator of population variance. Numerical results for the percent relative efficiency (PREs) in Table 2 reveals that the proposed estimator $\left(S_{p r}^{2}\right)$ has 153 percent gains in efficiency while the conventional ratio estimator of population variance by Isaki (1983) has 142 percent gains in efficiency; this shows that the proposed ratio estimator $\left(S_{p r}^{2}\right)$ is 11 percent more efficient than the Isaki 4] ratio estimator of population variance. Similarly, the proposed estimator $\left(S_{p r}^{2}\right)$ is 14 percent and 65 percent more efficient than the Bahl \& Tuteja [1] and the Solanki et al. [3] ratio-type estimators of population variance respectively. Also, in using the proposed ratio estimator, one will have 117 percent efficiency over the Kadillar \& Cingi [2] ratiotype estimator of population variance. Generally, the proposed ratio estimator fares better than every identified existing estimators of its family and has equal optimal efficiency with the regression estimator of population variance.

Table 2: MSEs and PREs for the estimators.

\begin{tabular}{|c|c|c|c|}
\hline New York: & New York: & New York: & New York: \\
\hline 1 & $S_{p r}^{2}$ & $2,045.09$ & 152.84 \\
\hline 2 & $S_{R E G}^{2}$ & $2,045.09$ & 152.84 \\
\hline 3 & $S_{R a t i o}^{2}$ & $2,200.83$ & 142.03 \\
\hline 4 & $S_{p r 2}^{2}$ & $2,243.21$ & 139.34 \\
\hline 5 & $S_{p r 4}^{2}$ & $3,571.96$ & 87.51 \\
\hline 6 & $S_{p r 3}^{2}$ & $8,776.33$ & 35.66 \\
\hline
\end{tabular}

\section{Conclusion}

Sequel to the discussion of results above, it is concluded that the proposed ratio estimator $\left(S_{p r}^{2}\right)$, fares better than every identified existing estimators of its family and has equal optimal efficiency with the general regression estimator $\left(S_{R E G}^{2}\right)$ which has always been preferred because of its efficiency. Therefore, the new ratio estimator of population variance in stratified random sampling should be preferred in practical situations by survey researchers as it provides consistent and more precise estimates of the population parameters of interest.

\section{References}

1. Bahl S, Tuteja RK (1991) Ratio and product type exponential estimator. Journal of Information and Optimization Sciences 12(1): 159-164.

2. Kadilar, Cingi H (2003) A study on the chain ratio-type estimator. Hacettepe Journal of Mathematics and Statistics 32: 105-108.

3. Solanki RS, Singh HP, Rathour A (2012) An alternative estimator for estimating the finite population mean using auxiliary information in sample surveys. ISRN Probability and Statistics, Article ID 657682.

4. Isaki CT (1983) Variance estimation using auxiliary information. Journal of the American Statistical Association 78(381): 117-123.

5. Khoshnevisan M, Singh R, Chauhan P, Sawan N, Smarandache F (2007) A general family of estimators for estimating population mean using known value of some population parameters. Far East Journal of Theoretical Statistics 22(2): 181-191.

6. Chaudhary MK, Singh R, Shukla RK, Kumar M, Smarandache F (2009) A family of estimators for estimating population mean in stratified sampling under non-response. Pakistan Journal of Statistics and Operation Research 5(1): 47-54.

7. Koyuncu N, Kadilar C (2009) Family of estimators of population mean using two auxiliary variables in stratified random sampling. Communications in Statistics-Theory and Methods 38(14): 2398-2417.

8. Vishwakarma GK, Singh HP, Singh S (2010) A family of estimators of population mean using multi-auxiliary variate and post-stratification. Nonlinear Analysis: Modelling and Control 15 (2): 233-253.

9. Tailor R, Sharma B, Kim J (2011) A generalized ratio-cum-product estimator of finite population mean in stratified random sampling. Communications of the Korean Statistical Society 18(1): 111-118.

10. Vishwakarma GK, Singh HP (2011) Separate ratio-product estimator for estimating population mean using auxiliary information. Journal of Statistical Theory and Applications 10(4): 653-664.

11. Malik S, Singh R (2012) Some improved multivariate-ratio-type estimators using geometric and harmonic means in stratified random sampling. ISRN Probability and Statistics, Article ID 509186.

12. Singh R, Audu A (2013) Efficiency of ratio estimator in stratified random sampling using information on auxiliary attribute. International Journal of Engineering and $\quad \mathrm{I} \mathrm{n} \mathrm{n} \mathrm{o} \mathrm{v}$ a $\mathrm{t} \mathrm{i} \mathrm{v}$ e Technology 2(1): 116-172.

13. Clement EP, Enang EI (2015) Calibration Approach Alternative Ratio Estimator for Population Mean in Stratified Sampling. International Journal of Statistics and Economics 16(1): 83-93.

14. Clement EP, Enang EI (2017) On the Efficiency of Ratio Estimator over the Regression Estimator.Communication in Statistics: Theory and Methods 46(11): 5357-5367.

15. Clement EP (2015) Calibration Approach Separate Ratio Estimator for Population Mean in Stratified Sampling. International Journal of Modern Mathematical Sciences 13(4): 377-384.

16. Clement EP (2016) An Improved Ratio Estimator for Population Mean in Stratified Random Sampling. European Journal of Statistics and Probability 4 (4): 12-17.

17. Clement EP (2017) Efficient Exponential Estimators of Population Mean in Survey Sampling. International Journal of Mathematics and Computation 28 (3): 94-106.

18. Clement EP (2017) Generalized Chain Ratio-Product Estimator for Estimating Population Mean With Auxiliary Variate. Elixir Statistics 106: 46471-46478. 
19. 1Clement EP (2017) Efficient Product-cum-Dual to Product Estimators of Population Mean in Systematic Sampling. Elixir Statistics 106: 46487-46493.

20. Agarwal MC, Sithapit AB (1995) Unbiased ratio type estimation Statistics and Probability Letters 25: 361-364.

21. Ahmed MS, Raman MS, Hossain MI (2000) Some competitive estimators of finite population variance multivariate auxiliary information. Information and Management Sciences 11(1): 49-54.

22. Upadhyaya LN, Singh HP (2001) Estimation of population standard deviation using auxiliary information. American Journal of Mathematics and Management Sciences 21(3-4): 345-358.

23. Upadhyaya LN, Singh HP (2006) Almost unbiased ratio and producttype estimators of finite population variance in sample surveys. Statistics in Transition (5): 1087-1096.

24. Arcos A, Rueda M, Martinez MD, Gonzalez S, Roman Y (2005) Incorporating the auxiliary information available in variance estimation. Applied Mathematics and Computation 160: 387-399.

25. Gupta S, Shabbir J (2008) Variance estimation in simple random sampling using auxiliary information. Hacettepe Journal of Mathematics and Statistics 37(1): 57-67.

26. Shabbir J, Gupta S (2006) On estimation of finite population variance. Journal of Interdisciplinary Mathematics 9(2): 405-419.

27. Kadilar C, Cingi H (2006) Ratio estimators for population variance in simple and stratified sampling, Applied Mathematics and Computation 173: $1047-1058$.

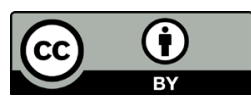

This work is licensed under Creative Commons Attribution 4.0 Licens

DOI: 10.19080/BBOAJ.2018.04.555659
28. Al-Jararha J, Al-Haj Ebrahem M (2012) A ratio estimator under general sampling design. Austrian Journal of Statistics 41(2): 105-115.

29. Bhushan S (2012) Some efficient sampling strategies based on ratio type, estimator. Electron J App Stat Anal 5(1): 74-88.

30. Tailor R, Sharma B (2012) Modified estimators of population variance in presence of auxiliary information. Statistics in Transition-New series 13(1): 37-46.

31. Singh HP, Solanki RS (2013) A new procedure for variance estimation in simple random sampling using auxiliary information. Statistical Papers 54(2): 479-497.

32. Subramani J, Kumarapandiyan G (2012) Variance estimation using quartiles and their functions of an auxiliary variable. International Journal of Statistics and Applications 2(5): 67-42.

33. Subramani J, Kumarapandiyan G (2013) Estimation of variance using known coefficient of variation and median of an auxiliary variable. Journal of Modern Applied Statistical Methods 12(1): 58-64.

34. Yadav SK, Kadilar C (2013) Improved Exponential type ratio estimator of population variance. Colombian Journal of Statistics 36(1): 145-152.

35. Cochran WG (1977) Sampling Techniques. (3 $3^{\text {rd }}$ edn), Wiley, New York, USA.

\section{Your next submission with Juniper Publishers}

will reach you the below assets

- Quality Editorial service

- Swift Peer Review

- Reprints availability

- E-prints Service

- Manuscript Podcast for convenient understanding

- Global attainment for your research

- Manuscript accessibility in different formats

( Pdf, E-pub, Full Text, Audio)

- Unceasing customer service

Track the below URL for one-step submission https://juniperpublishers.com/online-submission.php 